CERN LIBRARIES, GENEVA

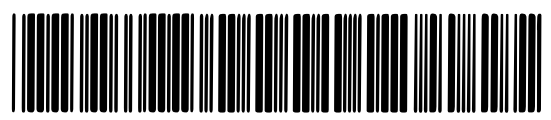

CM-P00064350

M. G. DOME

III

EUROPEAN ORGANIZATION FOR NUCLEAR RESEARCH

CERN ISR-BOM/81-15

MAGNETIC MEASUREMENTS ON THE ISR SUPERCONDUCTING QUADRUPOLES

by

L. Walckiers

Presented at the 7 th International Conference on Magnet Technology, Karlsruhe, Federal Republic of Germany

March 30 - Apri1 3, 1981

Geneva, Switzer 1 and

March 1981 


\section{MAGNETIC MEASUREMENTS ON THE ISR SUPERCONDUCTING QUADRUPOLES}

L. Walckiers

Abstract - The eight superconducting quadrupoles of the ISR high luminosity insertion were measured by the rotating coil method. This method allows an analysis of all harmonic components of the field.

In order to achieve magnetic measurements precise enough to check the quality of the magnet, a study of the errors inherent in such a measuring system was made. This paper describes how the measurement of the different harmonics of the field is affected by imperfections of the mechanical or electronic components of the measuring system. Following this study, the relative errors of the gradient are measured within $0.210^{-3}$ in the useful aperture, the absolute value of the quadrupole term is known within $0.510^{-3}$ as a function of current, and the magnetic axis is determined within 20 microns.

Results of the measurements show that by using the dodecapole correction winding, the relative error of the gradient integrated along the axis can be kept within the tolerance of $1 \%$ in the useful aperture of the magnets.

\section{INTRODUCTION}

In order to increase the luminosity in the CERN Intersecting Storage Rings, eight superconducting quadrupoles have been installed in intersection 8 during the autumn 1980 [1]. The nominal gradient is $43 \mathrm{~T} \mathrm{~m}^{-1}$ over magnetic lengths of $0.65 \mathrm{~mm}$ and $1.15 \mathrm{~m}$ for short and long magnets, respectively. The wann tube of the cryostats has an aperture of $173 \mathrm{~mm}$ and the beam optics specifies a useful horizontal aperture of $130 \mathrm{~mm}$.

In superconducting magnets, the field is defined by the position of the conductor rather than by the iron yoke. Consequently, the absolute value of the field obtained, as well as the quality of the field profile, is less reproducible between magnets than for magnets at room temperature. Furthermore, due to the high saturation of the yoke, the harmonics of the main field change appreciably with the value of the excitation. It is also difficult to define precisely the position of the magnetic axis with respect to the cryostat.

However, the quadrupoles have a strong effect on the parameters of the ISR proton beams. Thus, the specifications to be met are severe: a precision of $1 \%$ for the setting of the integrated gradient value, relative gradient errors below $1 \%$ in the useful aperture, and a positioning of the magnetic axis in the ISR ring with the greatest precision achievable. A sextupole winding is also mounted inside the quadrupole winding to correct the beam chromaticity, and a dodecapole winding to correct the field gradient profile in the useful range of current excitation.

Consequently, precise magnetic measurements were needed to measure the absolute value of the gradient, the field profile and the magnetic axis of all magnets.

Manuscript received March 30, 1981.

The author is with CERN, ISR Division, 1211 Geneva 23, Switzerland

\section{MEASUREMENT PRINCIPLE}

The rotating coil method [2] was judged to be the most appropriate for measuring magnets with this geometry. It gives a direct relationship between a twodimension-like magnetic potential and the complex coefficients $y_{n}$ obtained by the Fourier analysis of the integrated voltage measured across a coil rotating around the axis of the field.

The magnetic potential is given by:

$$
A(z)=\sum y_{n} z^{n},
$$

where $z=x+i y$ is the complex variable of the plane. The relation between this potential and the magnetic field integrated along the axis over a length $L$ is given by:

$$
\frac{d A}{d z}=\int_{L}\left(B_{y}+i B_{x}\right) d s \quad(T m),
$$

and the integrated voltage as a function of the angular position is:

$$
\mathrm{w}_{\mathrm{b}}(\theta)=\mathrm{R}_{\mathrm{e}}\left(\mathrm{K}_{\mathrm{n}} \mathrm{y}_{\mathrm{n}} \exp (i \theta)\right)
$$

The $K_{n}$ coefficients are calculated from the geometry of the measuring coil [2]. By connecting two coils in opposition with appropriate radii of rotation, it is possible to reduce $\mathrm{K}_{2}$ to zero. This method was used in order to measure the errors of the field gradient with a higher resolution (see section 4) than would be obtained with a single coil.

The relations between the radii of rotation are:

$$
\begin{aligned}
& \mathrm{R}_{\text {int, coil } 1}=1 / \sqrt{2} \quad \mathrm{R}_{\text {ext, }} \text { coil } 1 \\
& \mathrm{R}_{\text {ext, coil } 2}=\mathrm{R}_{\mathrm{int}, \operatorname{coi} 1} 1 \\
& \mathrm{R}_{\text {int, coil } 2}=0
\end{aligned}
$$

Three measuring coils placed along the magnetic axis enable the independent measurement of the ends and the central part of the quadrupoles. This is necessary to align the rotating tube with the magnetic axis and to take into account the end effects in order to calculate the longitudinal position of the magnetic centre. The coils measuring the ends are $600 \mathrm{~mm}$ long. The total measuring lengths are $1.8 \mathrm{~m}$ and $1.3 \mathrm{~m}$ for the long and the short magnets, respectively.

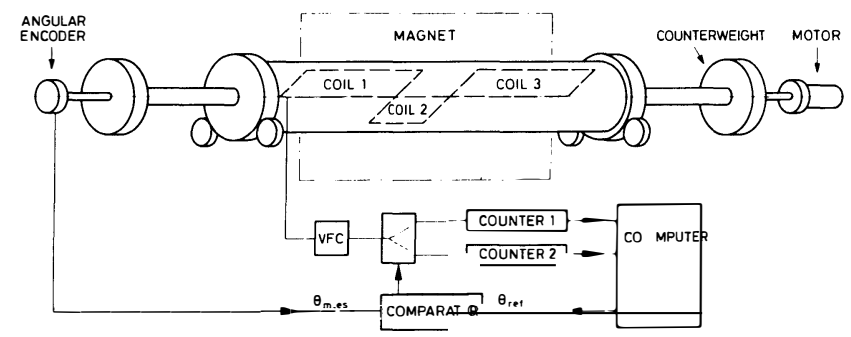

Fig. 1. Schematic layout of the measurement 


\section{ELECTRONIC SYSTEM AND DATA ANALYSIS}

The coil rotates continuously about its axis and a measurement is taken in one revolution. At one end, an electronic angular encoder is mounted. A voltage-tofrequency converter sends pulses to one of the two counters between two angular positions whilst the other counter containing the previously measured point is read by a computer. The number of points used is 512 per revolution. The measurements are then treated to take into account the calibration curve of the angular encoder and the offset of the electronic amplifier.

The computer gives an on-line value of the offcentre error and of the position of the zero of the angular encoder with respect to the magnetic plane. It can also print the absolute values of the harmonic coefficients, and plot curves of the gradient error. This gradient error is given by:

$$
\frac{\Delta G(z)}{G(o)}=\sum_{n>2} \frac{n(n-1)}{2} \frac{y_{n}}{y_{2}} z^{n-2} \text {. }
$$

\section{MEASUREMENTS OF THE GRADIENT PROFILE}

The accuracy of the measurements of the harmonic coefficients depends on the precision of the angular encoder, on the electronic equipment and on the accuracy of the rotation of the measuring coil about its axis.

The errors of the angular encoder are directly seen on the integrated voltage $\left(W_{b}(\theta)\right.$ in $\left.(3)\right)$. In practice, the relative increase of useful signal $\left(\mathrm{K}_{\mathrm{n}} \mathrm{y}_{\mathrm{n}} / \mathrm{K}_{2} \mathrm{y}_{2}\right)$ obtained by the difference method cancels these errors. The problems related to linearity, drift and noise of the electronic equipment are eliminated in the same way.

However, a displacement of the centre of rotation during a measurement is not compensated by this difference method. The ratio between this movement and the external radius of rotation of the measuring coil directly gives the accuracy obtainable. It can be calculated that, if the displacement $d$ of the centre of rotation with respect to its central position is of the form

$$
d(\theta)=C_{n} \cos n \theta \text {, }
$$

an error $E$ will be seen on the coefficients $n-1$ and $n+1$

$$
E\left(K_{n \neq 1} y_{n \pm 1}\right)=C_{n} y_{2}\left(R_{\text {ext }}-R_{\text {int }}\right) \text {, }
$$

where $R_{\text {ext }}$ and $R_{i n t}$ are the radii of rotation of the measuring coil. If one uses the compensating coil method (see (4)), then

$$
E\left(y_{n \pm_{1}}\right)=\left(1-\frac{2}{\sqrt{2}}\right) \frac{C_{n} y_{2}}{k_{n \pm 1}} R_{e x t, \operatorname{coil} 1} \text {. }
$$

Applying this relation, a variation of the deflection of the centre of the measuring coil (external radius $82 \mathrm{~mm}$ ) of \pm 4 ym gives an error in sextupole effect at a radial position of $65 \mathrm{~mm}$ of $1.510^{-4}$ in (5).

Consequently, the coils are mounted on tubes which are as rigid as possible, and the deflection at the centre is compensated by counterweights applied at the tube ends. The bearings are rectified within a few microns in order to ensure a precision of $10^{-4}$ for the measurement of $\Delta G / G_{O}$. This indicates that the precision of the system is a problem of rotational inaccuracy. The overall accuracy obtained in measuring the gradient errors is of the order of $210^{-4}$ in the useful aperture and can hardly be improved.

\section{POSITIONING OF THE MAGNETIC AXIS}

Since three coils are aligned in the measuring tube, it is possible to align this tube along the magnetic axis. The two components of the displacement, D, of the measuring coil compared to the magnetic axis is given by

$$
\mathrm{D}=\mathrm{y}_{1} / 2 \mathrm{y}_{2} \text {. }
$$

The most important error comes from the angular encoder. A torque about its axis leads to an error which is sinusoidal in nature, and which theoretically gives, for a quadrupole, an error in the dipole and sextupole terms. Consequently, the alignment between the axis of the encoder and the axis of rotation of the measuring tube is carefully carried out.

Nevertheless, the main limitation comes from the difficulty in transferring this measured axis on to the survey point mounted on top of the magnet cryostat. This can be done with a precision of no better than a few hundredths of a millimeter. This is, however, sufficient when compared to the precision of $0.1 \mathrm{~mm}$ when the magnet is finally installed in the ISR tunnel.

\section{MEASUREMENTS OF THE QUADRUPOLE COMPONENT}

To measure the absolute value of $y_{2}$ as a function of the excitation current, other coils are used having the internal radius of their windings aligned with the axis. The error $E\left(y_{2}\right)$ is then directly related to the precision with which the external radius of rotation of the coil is known.

$$
\frac{E\left(y_{2}\right)}{y_{2}}=2 \frac{E\left(R_{\text {ext }}\right)}{R_{\text {ext }}} .
$$

A measurement of this radius of rotation within $20 \mathrm{~mm}$ ensures a precision of $0.510^{-3}$ in the measurement of the gradient.

The same principle applies to the measurement of the sextupole term but the error due to the angular encoder must also be taken into account (see section 5).

\section{RESULTS}

The essential results of the magnetic measurements of the eight superconducting magnets have already been published [1]. They may be summarized as follows:

For the ISR superconducting quadrupoles, the components belonging to the quadrupole symmetry were measured to be equal to the values predicted by design calculations at different levels of current excitation. By an appropriate correction using the dodecapole winding, the quality of the gradient pattern is well within $1 \%$ over a horizontal aperture of $130 \mathrm{~mm}$. Typical values of the coefficients measured for a quadrupole and sextupole excited at their nominal values can be found in table 1 .

In addition, the results show that the position of the magnetic axis is reproduced, to within the accuracy of the measurement, after thermal cycling of the magnet to room temperature or following the transport of the magnets.

A magnet cycling procedure was also defined and verified in order to ensure a reproducibility of $10^{-4}$ for all field characteristics. 
Table 1

Harmonic coefficients $y_{n}(1)$ and their effects on the relative error of the gradient integrated along the axis at $65 \mathrm{~mm}$ from centre.

(Magnet SL6, I (4-pole) $=1600 \mathrm{~A}, \mathrm{I}(6$-pole $)=200 \mathrm{~A})$

\begin{tabular}{|c|c|c|c|c|c|}
\hline $\mathrm{n}$ & \multicolumn{3}{|c|}{$y_{n}$} & \multicolumn{2}{|c|}{$\begin{array}{c}\Delta \mathrm{G} / \mathrm{G}_{\mathrm{O}} \text { at } 65 \mathrm{~mm} \\
\times 10^{-3}\end{array}$} \\
\hline 2 & 24.9 & 0 & {$[\mathrm{~T}]$} & & \\
\hline 3 & 4.49 & 0.076 & {$\left[\operatorname{Tm}^{-1}\right]$} & 35.16 & 0.60 \\
\hline 4 & 0.11 & -0.46 & {$\left[\mathrm{Tm}^{-2}\right]$} & 0.12 & -0.47 \\
\hline 5 & -0.05 & -0.03 & {$\left[\operatorname{Tm}^{-3}\right]$} & -0.01 & 0 \\
\hline 6 & 63.0 & -0.6 & {$\left[\mathrm{Tm}^{-4}\right]$} & 0.68 & 0.01 \\
\hline 7 & -39.0 & 77.0 & {$\left[\operatorname{Tm}^{-5}\right]$} & -0.04 & 0.07 \\
\hline 8 & 13.0 & -585.0 & {$\left[\operatorname{Tm}^{-6}\right]$} & 0 & 0.05 \\
\hline 9 & $-2.010^{4}$ & $0.410^{4}$ & {$\left[\operatorname{Tm}^{-7}\right]$} & -0.15 & 0.03 \\
\hline 10 & $-1.0 \quad 10^{6}$ & $-2.0 \quad 10^{4}$ & {$\left[\mathrm{Tm}^{-8}\right]$} & -0.57 & 0 \\
\hline 14 & $-4.0 \quad 10^{9}$ & $-4.010^{8}$ & {$\left[\operatorname{Tm}^{-12}\right]$} & -0.09 & -0.01 \\
\hline 15 & $-1.410^{11}$ & $2.0 \quad 10^{9}$ & {$\left[\mathrm{Tm}^{-13}\right]$} & -0.22 & 0 \\
\hline 18 & $-6.010^{13}$ & $3.0 \quad 10^{12}$ & {$\left[\operatorname{Tm}^{-16}\right]$} & -0.04 & 0 \\
\hline
\end{tabular}

\section{CONCLUSION}

The measuring system described above enabled the measurement of the various field parameters to the required accuracy. This study nevertheless showed two limits in the precision of the rotating coil method for gradient measurement. The measurement of the gradient quality is limited to the order of $10^{-4}$ due to imperfections in the coil rotation system, and gradient values cannot be known to better than a few $10^{-4}$ due to the difficulties in defining the effective radius of rotation of the measuring coils.

\section{ACKNOWLEDGEMENTS}

This work was initiated by L. Resegotti and K. Henrichsen and their continuous help during the period of development of this system is greatly appreciated. Thanks are also due to $P$. Legrand, E. Magnani and P. Viret for the numerous tasks they carried out on electronic and mechanical components.

\section{REFERENCES}

[1] J. Billan et al., "The eight superconducting quadrupoles for the ISR high luminosity insertion", Proc. XIth Int. Conf. on High Energy Accelerators, CERN, Geneva, 1980

[2] C. Wyss, "A measuring system for magnets with cylindrical symmetry", Proc. 5th Int. Conf. on Magnet Technology, Frascati, 1975. 